\title{
A Direct Solution of Poisson's Equation by Generalized Sweep-Out Method*
}

\author{
By I. Hirota, T. Tokioka and M. Nishiguchi \\ Geophysical Institute, Tokyo University, Tokyo \\ (Manuscript received 21 November 1969, in revised form 6 February 1970)
}

\begin{abstract}
A direct method was designed to solve the Poisson equation for an arbitrarily shaped domain with the boundary conditions of Dirichlet or Neumann. The present method is essentially an extension of the one-dimensional "sweep-out method" to the two-dimensional case by using the matrix calculus.

The procedure of the present method consists of two parts, i.e., (1) the calculation of the residual maxtrix $R$ and its inverse matrix $R^{-1}$, and (2) the correction of the initial guess matrix $\Phi$ by the use of $R^{-1}$. Since $R$ depends only on the shape of the domain, this method is powerful in repeating to solve the equation for the same domain but with different boundary conditions and load functions.

An estimate of the number of arithmetic operations required for solving the equation indicates that the present method requires less machine time than those of other methods. Some remarks on the practical computations are also given.
\end{abstract}

\section{Introduction}

In dealing with the partial differential equations in many physical and geophysical problems, we are often faced with the necessity of solving boundary value problems such as Poisson-type and Helmholtz-type equation. A number of methods have been proposed for solving the equation numerically with the use of a finite difference approximation, and they may be grouped into two categories. One is the direct method, for instance, Tensor Product Method (TP) (Lynch et al., 1964) and Dimension Reduction Method (DRM) (Ogura and Charney, 1962; Ogura, 1969), and the other is the iterative method such as Richardson Method, Accelerated Liebmann Method (AL) and Alternating Direction Implicit Method (ADI) (See Forsythe and Wasow, 1960; Miyakoda, 1962).

From the viewpoint of users, it is of importance to know the merits and demerits of these methods on the speed of convergence, the computational stability, the applicability to the domain under consideration, the simplicity of computation procedure and so forth. For in-

* Division of Meteorology, Contribution No. 181. stance, AL method is very simple in scheme and applicable to any arbitrarily shaped domain, but it requires a considerable amounts of machine time. On the other hand, ADI method is somewhat complicated in scheme, though the convergence is rapid. DRM also requires less machine time, but has a demerit that it is not applicable to irregular domains.

The method proposed in the present paper for solving the boundary value problem is designed for the purpose of overcoming some of the defects mentioned above. It is well known that the onedimensional second-order ordinary differential equation with properly posed boundary conditions such as

$$
\begin{array}{lll}
\frac{d^{2} \phi}{d x^{2}}=\sigma(x) & \quad\left(x_{0} \leq x \leq x_{1}\right) \\
\phi=\phi_{0} & \text { at } & x=x_{0} \\
\phi=\phi_{1} & \text { at } & x=x_{1}
\end{array}
$$

can be solved directly (without iteration) with finite difference approximation by the "sweepout" method. The present method of this paper is essentially an extension of the sweep-out method to two-dimensional or multi-dimensional case. 


\section{The solution of Poisson's equation}

We will explain the procedure to solve the Poisson's equation with the boundary conditions of Dirichlet type. For simplicity, let us take a rectangular domain. As will be shown later, however, this restriction is easily relaxed.

\subsection{Residual matrix}

We divide the rectangular domain into $(M+1)$ $\times(N+1)$ meshes as is illustrated in Fig. 1 . First we set each element of the first row equal to zero except the $k$-th one $(1 \leq k \leq M)$, which is equal to unity. Hereafter we denote this rowvector as $\boldsymbol{e}_{k}$.

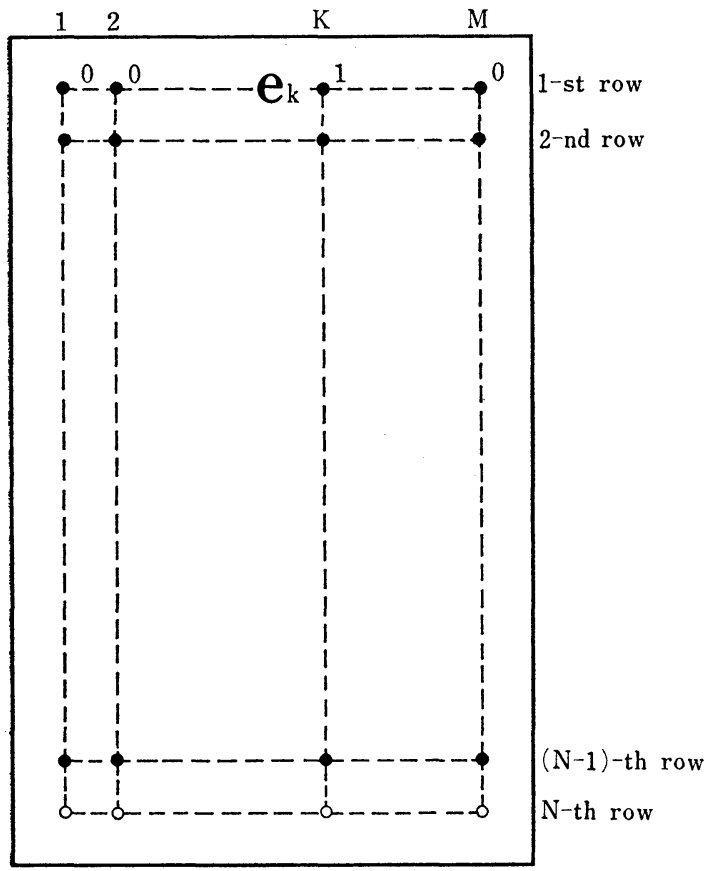

Fig. 1. Mesh-points in a rectangular domain for solving the Poisson's equation with the boundary condition of Dirichlet-type.

Finite difference form of the Poisson's equation is written as

$$
\begin{aligned}
& \left(\phi_{i+1, j}+\phi_{i-1, j}+\phi_{i, j_{+}}\right. \\
& \left.\quad+\phi_{i, j-1}-4 \phi_{i, j}\right) /(\Delta h)^{2}=\sigma_{i, j}
\end{aligned}
$$

by using the ordinary 5-point difference approximation. $\Delta h$ is a mesh size and in the following discussion we set $\Delta h$ equal to unity to avoid unnecessary confusions. If the equation (2-1) is applied to the first row on which $\phi$ is equal to $\boldsymbol{e}_{k}$, together with the conditions

$$
\begin{aligned}
& \sigma_{i, j}=0 \quad\left(\begin{array}{l}
i=1,2, \cdots \cdots M \\
j=1,2, \cdots \cdots N
\end{array}\right) \\
& \text { and } \\
& \phi=0 \text { on the boundary, }
\end{aligned}
$$

we can determine the value of $\phi$ on the second row. By repetition of this procedure, the value of $\phi$ is obtained at every meshpoint of the domain. On the $N$-th row, however, the equation (2-1) does not hold and there exist residuals, since the value of $\phi$ on the $N$-th row is determined from the equation applied to the $(N-1)$-th row. We denote this residual vector as $\boldsymbol{R}_{k}$. By varying $k$ from 1 to $M$, a set of residual vectors $\boldsymbol{R}_{k}(k=1$, $2, \cdots \cdots M)$ is obtained corresponding to $\boldsymbol{e}_{k}(k=1$, $2, \cdots \cdots M)$.

We define a matrix $R$ as

$$
R=\left(\begin{array}{c}
\boldsymbol{R}_{1} \\
\boldsymbol{R}_{2} \\
\vdots \\
\boldsymbol{R}_{M}
\end{array}\right)
$$

which we name "residual matrix". This matrix depends only on the shape of the domain and the way to select $\boldsymbol{e}$-vector.

\subsection{Solution of equation}

Let's treat the equation (2-1) together with the prescribed boundary values $\phi$ and load function $\sigma$. If we have an initial guess of $\phi$ on the first row and repeat the same procedure as was practiced in the preceding subsection to obtain $\boldsymbol{R}_{k}$, an initial guess matrix $\Phi^{0}$ (with $M N$ elements) and a residual vector $\widetilde{\boldsymbol{R}}$ on the $N$-th row are obtained.

It is evident that $\phi$ is the exact solution of $(2-1)$ if the residual vector $\widetilde{\boldsymbol{R}}$ is zero. Therefore our purpose is to find a correction matrix which has a residual vector $-\widetilde{\boldsymbol{R}}$ on the $N$-th row under the conditions of zero load function and zero boundary values.

Assume that $\boldsymbol{R}_{k}(k=1,2 \cdots \cdots M)$ are linearly independent, i.e.,

$$
\operatorname{Det} R \neq 0 \text {. }
$$

Then the vector $\widetilde{\boldsymbol{R}}$, which consists of $M$ elements, will be uniquely represented by the linear combination of $\boldsymbol{R}_{k}$, i.e.,

$$
\widetilde{\boldsymbol{R}}=\sum_{k=1}^{M}\left(-\delta \phi_{k}\right) \boldsymbol{R}_{k}
$$

or

$$
\widetilde{\boldsymbol{R}}=-\delta \boldsymbol{\phi} \cdot R
$$


where $\delta \phi$ is a row vector with $M$ elements. $\delta \phi$ can be obtained under the assumption (2-3) as follows;

$$
\delta \phi=-\widetilde{\boldsymbol{R}} \cdot R^{-1},
$$

where $R^{-1}$ is the inverse matrix of $R$. Let's set $\delta \phi$ on the first row and repeat the same procedure as that to obtain $\Phi^{0}$, but for zero load function $\sigma$ and zero boundary values. Then we have a correction matrix $\Delta \Phi$. Since $\delta \phi$ is rewritten as $\delta \phi=\sum_{k=1}^{M} \delta \phi_{k} \boldsymbol{e}_{k}$, it follows from (2-4) that the residual vector corresponding to $\Delta \Phi$ is $\sum_{k=1}^{M} \delta \phi_{k} \boldsymbol{R}_{k}=-\widetilde{\boldsymbol{R}}$ on the $N$-th row. If we add $\Delta \Phi$ to $\Phi^{0}$, i.e., $\Phi=\Phi^{0}$ $+\Delta \Phi, \Phi$ satisfies the equation (2-1) under the prescribed boundary conditions and the load function $\sigma$. Thus we have obtained the solution.

The remaining problem is whether the assumption $(2-3)$ is always valid or not. We will give a proof of (2-3) in the following section.

\section{Proof of Det $R \neq 0$}

Let us consider the following problem;

$$
\begin{gathered}
\phi_{i, j+1}+\phi_{i, j-1}+\phi_{i+1, j}+\phi_{i-1, j}-4 \phi_{i, j}=\sigma_{i, j} \\
\sigma_{i, j}=0 \quad\left(\begin{array}{l}
1 \leq i \leq M \\
1 \leq j \leq N-1
\end{array}\right) \\
\sigma_{i, N} \neq 0 \\
\phi=0 \text { on the boundary }
\end{gathered}
$$

Corresponding to Green's theorem in continuous form, this set of equation also has a unique solution $\Phi$. This is directly confirmed from the non-vanishing of $\operatorname{Det} A$ (cf. Karlqvist, 1952), where $A$ is the matrix obtained from the coefficients of (3-1) when the equation is rewritten as

$$
A \cdot \Phi=(\sigma) .
$$

We assume that $\boldsymbol{R}_{k}(k=1,2, \cdots \cdots M)$ is linearly dependent, then for $₫ k_{0}\left(1 \leq k_{0} \leq M\right), \quad \boldsymbol{R}_{k_{0}}$ is written as

$$
\boldsymbol{R}_{k 0}=\sum_{k=1}^{M} a_{k} \boldsymbol{R}_{k},
$$

where $\Sigma^{\prime}$ denotes a summation except for $k=k_{0}$. Consider the problem (3-1) with $\left(\sigma_{i, N}\right)=\boldsymbol{R}_{k}$. The uniqueness of the solution of (3-1) indicates that the first row of the solution is $\boldsymbol{e}_{k}$. Accordingly, if we set $\left(\sigma_{i, N}\right)=\sum^{\prime} a_{k} \boldsymbol{R}_{k}$ then the first row of the solution becomes $\Sigma^{\prime} a_{k} \boldsymbol{e}_{k}$. Therefore, owing to the assumption (3-2), the row-vector $\boldsymbol{e}_{k 0}$ should be represented by

$$
\boldsymbol{e}_{k 0}=\Sigma^{\prime} a_{k} \boldsymbol{e}_{k},
$$

which is apparently incorrect from the definition of $\boldsymbol{e}$ in section 2-1. This inconsistency comes from the incorrect assumption (3-2). Therefore it is concluded that $\operatorname{Det} R \neq 0$.

\section{Application to Neumann's problem}

We have discussed Dirichlet's problem. In this section an attempt is made to apply the present method to Neumann's problem. Assume that $\nabla^{2} \phi=\sigma$ and $\partial \phi / \partial n=\xi$ are given on the boundary, where $n$ denotes the direction normal to the boundary. We consider a rectangular domain for the sake of simplicity (see Fig. 2). Poisson's

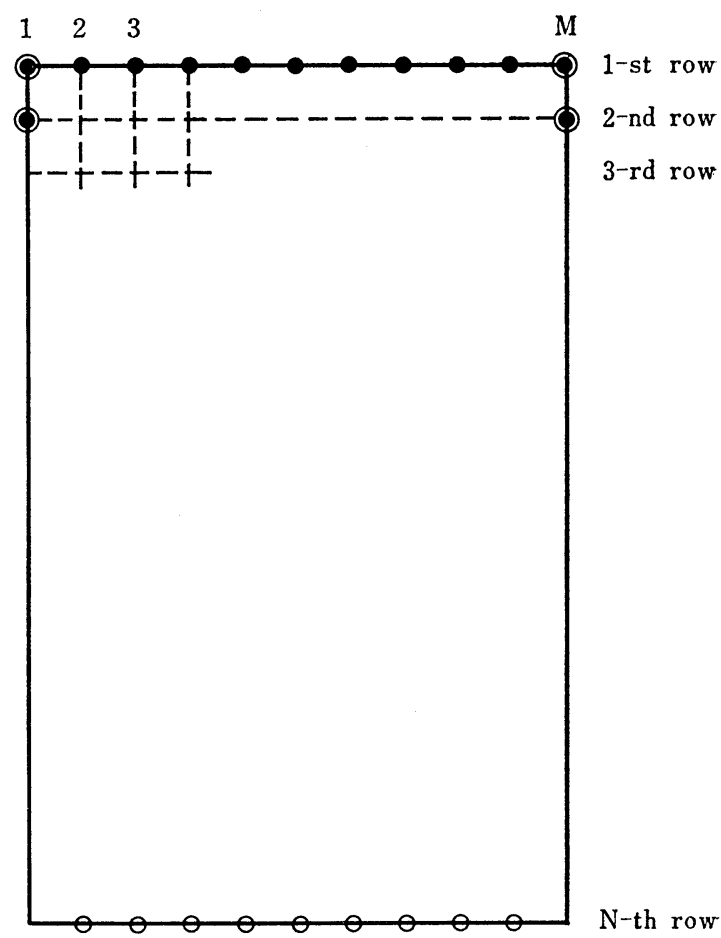

Fig. 2 Same as Fig. 1 but for the boundary condition of Neumann-type.

equation and boundary condition are expressed by the following finite difference form on the boundary mesh-point denoted by solid circle in Fig. 2.

$$
\begin{gathered}
\phi_{i, 0}+\phi_{i, 2}+\phi_{i+1,1}+\phi_{i-1,1}-4 \phi_{i, 1}=\sigma_{i, 1} \\
\phi_{i, 2}-\phi_{i, 0}=2 \xi_{i, 1}
\end{gathered}
$$

where $\phi_{i, 0}$ is a value of an artificial point outside of the domain. Eliminating $\phi_{i, 0}$ between (4-1) and (4-2), we have 


$$
2 \phi_{i, 2}+\phi_{i+1,1}+\phi_{i-1,1}-4 \phi_{i, 1}=\sigma_{i, 1}+2 \xi_{i, 1}
$$

Similar equations are derived on the side boundaries. If we set an initial guess on solid cir les, values on the point denoted by double circles are determined by conventional means;

$$
\left.\begin{array}{l}
\phi_{2,1}-\phi_{1,1}=\xi_{1}, 2 \\
\phi_{1,2}-\phi_{1},{ }_{1}=\xi_{2,1}
\end{array}\right\}
$$

Then $\phi_{i, 2}(2 \leq i \leq M-1)$ are uniquely determined from (4-3). $\phi_{1,3}$ and $\phi_{M}, 3$ are also determined from the boundary equation corresponding to (4-3). After the repetition of the similar procedure, we have the values of $\phi$ that satisfy $\nabla^{2} \phi=\sigma$ in the interior and boundary conditions except at the $N$-th row. On the lower boundary meshpoints denoted by white circles, there remain residuals $\widetilde{\boldsymbol{R}}$.

On the other hand, the procedure to calculate the residual matrix $R$ in this case is following. First we set $\boldsymbol{e}_{k}$-vector $(2 \leq k \leq M-1)$ on the solid circle points, and setting $\sigma$ and $\xi$ equal to zero, we have residual vector $\boldsymbol{R}_{k}$ on the $N$-th row.

Using the matrix $R$ defined by (2-2), we have a direct solution of this case in a similar way as was described in $2-2$.

\section{Application to domain of irregular shape}

It is easily found that the present method is also applicable to the case of an irregular domain, provided that the boundary condition is properly posed. One of the most important points of the present method is that we can choose $M$ meshpoints on which $\boldsymbol{e}_{k}$ is defined and corresponding $M$ mesh-points on which $\boldsymbol{R}_{k}$ is uniquely determined. The number of the mesh-points $M$, as well as their locations in the domain, depends upon the shape of the domain under consideration, and there remain some freedoms to choose. Therefore the arrangement of the mesh-points on which $\boldsymbol{e}$ and $\boldsymbol{R}$ are defined should be made from the viewpoints of the actual computation.

Fig. 3 shows examples of the arrangement of the mesh-points in irregularily shaped domains, where $\boldsymbol{e}$-vector and $\delta \boldsymbol{\phi}$ are defined on solid circles and $\boldsymbol{R}_{k}$ and $\widetilde{\boldsymbol{R}}$ are determined on white circles. No change is required in the procedure to solve the equation and the proof of Det $R \neq 0$ mentioned earlier. It is further noted that the present method is applicable not only to the grid system on the Cartesian coordinate but also to that on arbitrary coordinate systems for the respective finite difference formula of the Laplacian operation.

\section{Estimate of the number of numerical operations}

A rough estimate is made in this section for the number of numerical operations required to solve the equation by the present method. We confine ourselves to estimate only the number of multiplications and divisions, since the machine time required for additions and subtractions can be neglected practically.

The procedure of the present method is divided into two stages:

(1) To construct the matrix $R$ and to calculate the inverse matrix $R^{-1}$.

(2) To construct the residual vector $\widetilde{\boldsymbol{R}}$ and to calculate the correction vector $\delta \phi=-\tilde{\boldsymbol{R}} \cdot R^{-1}$ and the solution $\emptyset$.

When we consider the domain spanned by a $N \times N$ square mesh, the number of operations required for performing the procedure is roughly estimated corresponding to the two stages as the

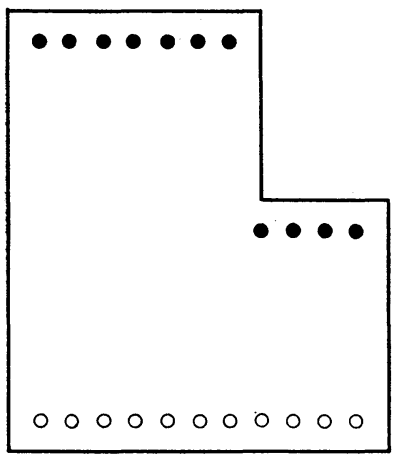

(a)

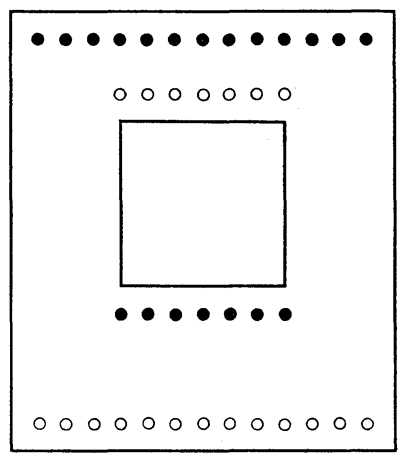

(b)

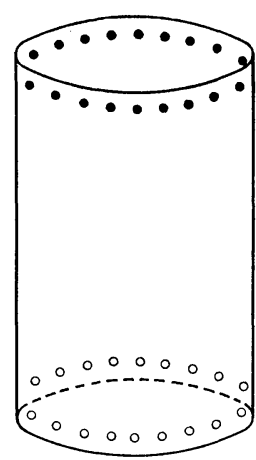

(c)

Fig. 3 Examples of irregular shape. $\boldsymbol{e}_{k}$ is defined on solid circles and $\boldsymbol{R}_{k}$ on white circles. 
order of (1) $2 N^{3}$ and (2) $3 N^{2}$ respectively. When the domain is a rectangular spanned by $M \times N$ as was illustrated in Fig. 1, they are reduced to (1) $M^{2} N+M^{3}$ and (2) $2 M N+M^{2}$ respectively.

It is found that the most of the operations is included in the stage (1), since $2 N^{3} \gg 3 N^{2}$ for the large value of $N$. However, if we have prepared in advance the inverse matrix $R^{-1}$ which depends only on the shape of the domain, the number of operations required for solving the equation for a given boundary condition and a load function becomes only of the order of $3 N^{2}$. Therefore it should be emphasized that the present method is powerful in repeating to solve the equation for the same domain but with different boundary conditions and load functions. In practice, we often meet this requirement in carrying out the time integration of a set of equations of fluid motion.

Ogura (1969) has estimated the number of operations required in DRM and made a comparison with those of other methods. According to the Table 2 of Ogura (1969), the number of multiplications and divisions required to solve Poisson's equation is of the order of $5 N^{3} \log N$ (AL), $18 N^{2} \log ^{2} N$ (ADI), $8 N^{2} \log _{2} N$ (TP) and $4 N^{2} \log _{2} N$ (DRM) respectively. Compared with the estimates for these methods, $3 N^{2}$ (present method) is very small, showing that it requires less machine time.

\section{A remark on the actual computation}

A remark is given on the accuracy of the solutions from the viewpoint of the actual computation. There are no difficulties to obtain the direct solution of the Poisson's equation by the present method theoretically, but in performing the numerical calculations one may meet a difficulty in that the accuracy of $R^{-1}$ is insufficient to give the correction vector $\delta \phi$. Generally speaking, when the domain is spanned by a $N \times N$ mesh, the order of the elements of $R$ and $\widetilde{\boldsymbol{R}}$ is roughly estimated as;

$$
\begin{array}{lll}
R \sim 0\left(4^{N}\right) & \text { for } & \boldsymbol{e} \sim 0\left(10^{\circ}\right) \\
\widetilde{\boldsymbol{R}} \sim 0\left(4^{N}\right) & \text { for } & \delta \boldsymbol{\phi} \sim 0\left(10^{\circ}\right)
\end{array}
$$

Therefore, in order to have the correction vector $\delta \phi$ accurately from the relation

$$
\delta \phi=-\tilde{\boldsymbol{R}} \cdot R^{-1},
$$

high accuracy of $R^{-1}$ should be required for large
$N$. Then it is suggested that when $N$ is large the computation of the inverse matrix $R^{-1}$ is made by the use of "double precision" in a computer.

When $R^{-1}$ has a small error, however, we have a way to overcome this deficiency by the repetition of the procedure (7-1). Suppose that $\phi^{(\tau)}$ is the $\tau$-th guess on the first row, $\widetilde{\boldsymbol{R}}^{(\tau)}$ the corresponding $\tau$-th residual vector on the $N$-th row, and $\delta \phi^{(\tau)}$ the $\tau$-th correction vector, then we have

$$
\delta \phi^{(r)}=-\widetilde{\boldsymbol{R}}^{(r)} \cdot R^{-1} .
$$

Since the series $\delta \phi^{(\tau)}(\tau=1,2, \cdots \cdots)$ converges to a small value under the limit of the error of $R^{-1}$, $\phi^{(\tau)}$ also converges to a certain level, that is, an approximate solution of the equation.

An example of the convergence for the case of $M=9$ and $N=19$ is shown in Fig. 4, where the

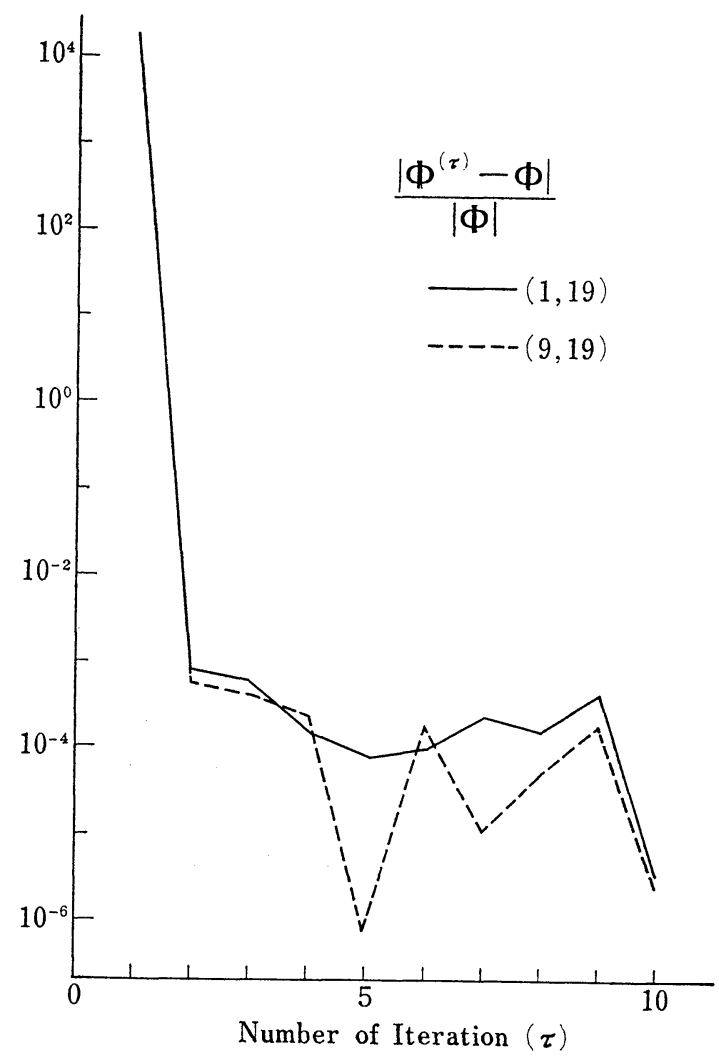

Fig. 4 An example of the convergence for the case of $M=9$ and $N=19$.

abscissa indicates the number of the repetition of (7-1) and the ordinate the relative error in logarithmic scale. In this case, $\left|\left(\phi^{(\tau)}-\phi\right) / \phi\right|$ on the 19-th row reaches to $0\left(10^{-4}\right)$ at $\tau=2$ and further convergence cannot be attained. When 
either the error of $R^{-1}$ is sufficiently small or the initial guess of $\phi$ does not differ so much from the exact solution, the repetition will be saved.

Another way to overcome the deficiency is to arrange the mesh-points, on which $\boldsymbol{e}$ and $\boldsymbol{R}$ are defined, in such a manner that the order of the elements of $R$ and $\tilde{\boldsymbol{R}}$ becomes smaller than $0\left(4^{N}\right)$. Fig. 5 shows an example of the arrangement of the mesh-points, though in this case the dimension of the matrix $R$ is enlarged from $(M, M)$ to $(2 M, 2 M)$.

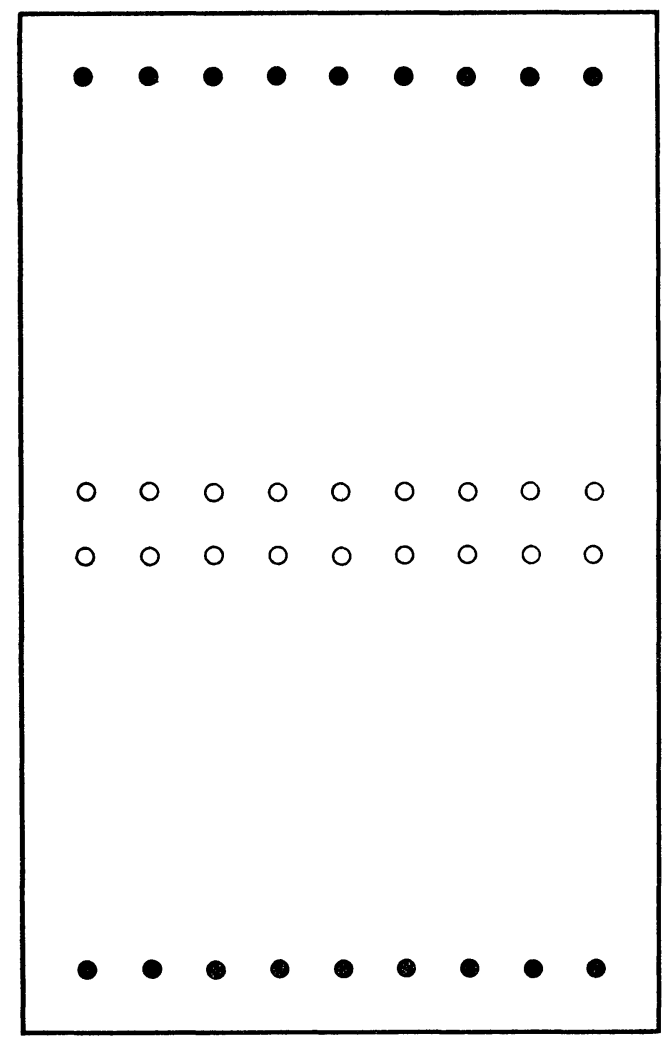

Fig. 5 An example of the arrangement of $\boldsymbol{e}_{k}$ and $\boldsymbol{R}_{k}$ for a very long rectangular domain.

\section{Summary}

A method for solving the Poisson's equation was proposed. The procedure of the present method is essentially an extension of the so-called "sweep-out method", and so we tentatively name the method "Generalized Sweep-out Method (GSM)".

The characteristics of the present method are summarized as follows:

(1) GSM is applicable to any irregularily shaped domain with the boundary conditions of
Dirichlet-type and Neumann-type.

(2) The procedure of GSM is divided into two stages. If the first stage has been carried out in advance, GSM is powerful in repeating to solve the equation for the same domain but with different boundary conditions and load functions.

(3) Concerning the number of operations required for solving the equation, the second stage of GSM requires only $3 N^{2}$ which is very small compared with those of other methods so far proposed.

(4) When $N$ is large, the requirement on the accuracy of the inverse matrix $R^{-1}$ becomes severe. However, we can overcome the deficiency by making some modifications of GSM.

It should be further noted that GSM is applicable not only to the Poisson's equation but also to the Holmholtz's equation free from the Collatz condition. Finally, it is also suggested that GSM can be extended from the case of twodimension to that of three-dimension, where the matrix calculus is replaced by the tensor calculus.

\section{Acknowledgments}

The present authors wish to express their hearty thanks to Prof. M. Yanai for his encouragement throughout this work. They are deeply indebted to Dr. K. Miyakoda of GFDL, ESSA, for his valuable comments on the first manuscript of this paper. This research was supported by Funds of Scientific Research from the Ministry of Education. Thanks are extended to Miss I.T. Tamura for typing the manuscript.

\section{References}

Forsythe, G.E., and W.R. Wasow, 1960: Finitedifference methods for partial differential equations. John Wiley \& Sons Inc., New York, 444 pp.

Karlqvist, O., 1952: Numerical solution of elliptic difference equations by matrix methods. Tellus, 4 , 374-384.

Lynch, R.E., J. Rice, and D.H. Thomas, 1964: Direct solution of partial differential equations by tensor product method. Num. Math., 6, 185-199.

Miyakoda, K., 1962: Contribution to the numerical weather prediction-computation with finite diference- Japanese Jour. Geophys., 3, No. 1, 76-190.

Ogura, Y., and J.G. Charney, 1962: A numerical model of thermal convection in the atmosphere. Proc. Intern. Symp. Numer. Wea. Pred., Tokyo, 431-451. 


\title{
一般化された掃出し法による Poisson 方程式の直接解法
}

\author{
廣田 勇・時 岡 達 志・西口三登志 \\ (東京大学理学部地球物理学教室)
}

Poisson 方程式を任意の形の領域で Dirichlet 型及び Neumann 型境界条件のもとで直接解く方法を考案した. この方法は, 二階常微分方程式を解く際に用いられる「掃出し法」を, 行列演算を用いて二次元以上の場合に拡張し たものである.

この解法の手続きは二つの段階に分けられる. 即ち, (1) residual matrix $R$ 及びその逆行列 $R^{-1}$ を求めること, (2) initial guess matrix $\Phi$ を $R^{-1}$ を用いて修正すること,である. 第一の段階は領域の形のみによるから, 同一領 域で異った境界条件と荷重函数に対し繰り返し方程式を解く場合にこの方法は有力である. 解法に要する演算の回数 を当ってみると, 計算時間は他の方法に比して少くて済むことがわかる．この方法を用いる場合の実際的な注意につ いても触れる。 